\title{
Effects of habitat-modifying invasive macroalgae on epiphytic algal communities
}

\author{
Emily Jones ${ }^{1,2, *}$, Carol S. Thornber ${ }^{1}$ \\ ${ }^{1}$ Department of Biological Sciences, University of Rhode Island, Kingston, Rhode Island 02881, USA \\ ${ }^{2}$ Bodega Marine Laboratory, University of California Davis, Bodega Bay, California 94923, USA
}

\begin{abstract}
Although invasive species can negatively impact communities via processes such as resource competition, they may also add new resources that facilitate the distribution and/or abundance of other organisms. In rocky intertidal systems, many benthic macroalgae compete for primary substrate, while providing secondary substrata to which sessile organisms can attach. Using field surveys and laboratory experiments, we investigated algal host-epiphyte dynamics in a New England rocky intertidal system. First, we compared the composition, abundance, richness, and diversity of epiphytes on 2 invasive macroalgal species, Codium fragile ssp. tomentosoides and Grateloupia turuturu, to 2 native macroalgal species, Chondrus crispus and Fucus vesiculosus. We found significant differences among epiphyte communities, as all macroalgal hosts supported different assemblages of species. While epiphyte richness and diversity were generally low on F. vesiculosus and G. turuturu year-round, they were low on C. crispus during the winter only. In contrast, epiphyte richness and diversity on $C$. fragile remained high throughout the year, suggesting that $C$. fragile plays an important role for supporting epiphytes during the cooler months. Second, we examined the relationship between epiphytes and a common herbivorous snail, Lacuna vincta. The abundance of juvenile $L$. vincta was positively correlated with 1 of the 2 most common epiphyte species, Neosiphonia harveyi. However, $L$. vincta showed a significant consumption preference for the other abundant epiphyte, Ceramium virgatum, in laboratory assays. Our results suggest that epiphyte facilitation by these invasive algal hosts is not only seasonally important for maintaining species richness and diversity, but may also provide both food and habitat for higher trophic levels.
\end{abstract}

KEY WORDS: Invasive macroalgae $\cdot$ Epiphyte $\cdot$ Habitat-modification $\cdot$ Codium fragile $\cdot$ Grateloupia turuturu $\cdot$ Lacuna vincta

Resale or republication not permitted without written consent of the publisher

\section{INTRODUCTION}

It is well known that the addition of invasive species into communities can lead to declines in native species richness and diversity, and most scientific research has focused on the negative impacts of biological invasions (Williamson 1996, Wilcove et al. 1998, Parker et al. 1999, Sanders et al. 2003). Despite the recent interest in positive interactions and their importance for structuring ecological communities, less attention has been focused on the potential positive interactions that may occur between native species and invaders (Bruno et al. 2005, Rodriguez 2006). As new ecological interac- tions are established with the introduction of an invasive species, both competition and facilitation may be important mechanisms for controlling the species composition within communities. Thus, while some native species may be displaced in the presence of an invader, the abundance and diversity of other native species may be enhanced through these new interactions (Posey 1988, Crooks 1998, 2002). In addition, the consequences of these interactions may spread across trophic levels to affect the greater community (Rodriguez 2006)

In rocky intertidal systems, one of the most limiting resources for both plants and animals is space (Dayton 
1971). Some marine organisms, however, can act as foundation species (sensu Dayton 1972), thereby increasing substrate heterogeneity and the area available for settlement. Reviews have emphasized the critical role that these habitat-forming species can play in facilitating associated organisms (Stachowicz 2001, Bruno et al. 2003). For example, many benthic macroalgae serve as both primary spaceholders in communities, competing for resources such as space, as well as a secondary substratum, creating a physical structure to which other organisms can attach. Species that rely on secondary substrate, such as epiphytic algae, epibiotic/sessile invertebrates, and many other microfauna, may thus benefit from the presence of a habitatforming introduced species (Crooks 2002, Rodriguez 2006). Although many ecological studies have examined how native benthic algal species and associated invertebrates are displaced by these invasions (e.g. Levin et al. 2002, Casas et al. 2004, Sanchez et al. 2005, Scheibling \& Gagnon 2006), ecologists have only recently begun to examine how the direct addition of new substrate by invasive species influences the abundance, richness, and diversity of algal epiphytes and/or sessile invertebrates (e.g. Wernberg et al. 2004, Wikström \& Kautsky 2004, Schmidt \& Scheibling 2006, Strong et al. 2006).

In rocky intertidal areas of southern New England, large perennial macroalgal species richness is low, with communities primarily composed of a few coarse (tough/thick) algae, such as Chondrus crispus, Fucus spp., and Ascophyllum nodosum (Villalard-Bohnsack et al. 1988). These species dominate the primary substrate, often covering nearly $100 \%$ of the available rock space (Menge 1976, Lubchenco 1980). Most of the native algal diversity in New England is represented by small, filamentous, or fast-growing ephemeral species, $>60 \%$ of which have the potential to grow epiphytically (Jones 2007). In addition, in areas where bare rock space is available, many of these intertidal annual species fail to successfully colonize or exploit the resource, perhaps due to shading, sedimentation, grazing, or wave exposure (Menge 1976). It appears that secondary substrate suitable for settlement may be essential for many algal species and for maintaining high algal diversity in this area.

Rocky shorelines of New England have been invaded by 2 large macroalgal species: Codium fragile ssp. tomentosoides (van Goor) P. C. Silva, in 1957 (Bouck \& Morgan 1957) and Grateloupia turuturu Yamada, in 1994 (Villalard-Bohnsack \& Harlin 1997). These species have negatively impacted intertidal and subtidal algal communities by indirectly displacing native benthic algal species (Harris \& Tyrell 2001, Levin et al. 2002). However, these species both provide macroalgal substrata that are different from the native species. For example, C. fragile has soft, spongy tissue and cylindrical branches that rise vertically in the water column. Its structure may provide a high surface area for settlement, crevices where algal recruits are potentially protected, and increased light availability for epiphytes that are raised closer to the surface during submersion. G. turuturu, having a large fleshy blade with a silky texture, also provides a large surface area for attachment. In addition, unlike other intertidal species with similar morphology, G. turuturu persists as a blade for 10 to 12 mo of the year (Harlin \& Villalard-Bohnsack 2001). These general characteristics of algal size, longevity, and surface relief, as well as cell wall structure and the presence of secondary metabolites, have all been noted as important for the presence or absence of epiphyte growth (Seed \& O'Connor 1981, Schmitt et al. 1995, Dawes et al. 2000, Steinberg et al. 2002). Over 20 epiphyte species have been identified on C. fragile in the Gulf of Maine and Northwest Atlantic (Wilson 1978, Trowbridge 1999, Mathieson et al. 2003), and at least 13 species have been identified on G. turuturu in Rhode Island (Villalard-Bohnsack \& Harlin 2001). However, while these studies have identified and quantified these epiphytes, no published studies have determined how these 2 invasive host species affect epiphyte composition in relation to other New England algal hosts.

Epiphyte species supported by invasive algal hosts may also provide resources for higher trophic levels. Many mesograzers, such as isopods, amphipods, and small gastropods, use epiphytic algae for food and/or habitat (Jernakoff et al. 1996, Pavia et al. 1999, OravKotta \& Kotta 2004). Therefore, the presence of epiphytic algae on less palatable host species (e.g. Chavanich \& Harris 2002) may create a more suitable environment for mesograzers than hosts devoid of epiphytic algae. Studies examining how the positive interactions created among host and epiphyte species may, in turn, influence herbivorous epiphyte grazers are lacking.

In the present study, we investigated the influences of 2 habitat-forming invasive algal species, Codium fragile ssp. tomentosoides and Grateloupia turuturu, on epiphytic algal diversity, richness, abundance, and community structure. We also examined abundance and feeding preference relationships between common epiphyte species and the herbivorous snail Lacuna vincta. Using field surveys and experiments, we asked the following: Does algal epiphyte abundance, richness, diversity, and composition differ between native and invasive host species? Do secondary substrate relief and host height affect algal epiphyte abundance, richness, and diversity? Do native and invasive species facilitate algal epiphyte species that $L$. vincta differentially associate with and consume? 


\section{MATERIALS AND METHODS}

Site characteristics. Field surveys were conducted in the low intertidal at Brenton Point State Park in Newport, Rhode Island, USA. This open coast site is composed of large rocky benches that extend into the subtidal zone. Bare space is low $(<15 \%)$, and the primary substrate is dominated by large perennial algal species. One of the most common low intertidal gastropods at this site is Lacuna vincta Montagu (E. Jones pers. obs.), a small $(<3 \mathrm{~mm})$ herbivorous snail.

Surveys of algal epiphyte abundance, richness, and diversity. In order to quantify algal epiphyte abundance, richness, and diversity on invasive versus native host species, we conducted repeated field surveys of 4 host species (natives: Fucus vesiculosus Linnaeus and Chondrus crispus Stackhouse; invasives: Codium fragile and Grateloupia turuturu; subsequently referred to by genus alone) from June 2005 to September 2006. All collections were made during low tides $<0.2$ MLLW. We randomly collected 10 replicates of each host species every month during the spring and summer, and bimonthly during the fall and winter. In order to cover the large range of habitat heterogeneity, we used random number generation to select each sample from a unique $x, y$ coordinate along a pair of meter tapes: one $10 \mathrm{~m}$ tape running parallel to the shore at the upper boundary of species overlap and one $6 \mathrm{~m}$ tape running perpendicular to the shore. At each random $(x, y)$ point of the transect, all upright fronds and holdfast of the alga (1 replicate) located within $1 \mathrm{~cm}^{2}$ were removed from the primary substrate (rock); thus, a replicate could be the entire thalli or a portion of a large individual (or ramet). Samples were collected twice for each sampling month, with 5 replicates of a host collected each time. Transects for each sampling date were at least $20 \mathrm{~m}$ apart from each other.

We took all samples back to the laboratory. Initial photographs of each sample were taken as a voucher of epiphyte cover; then, we removed, sorted, and identified all epibiotic organisms. Because few sessile invertebrates were present in our samples $(<5 \%$ total biomass) and microscopic algae were difficult to quantify due to their small size and/or fragility of cell aggregations (benthic diatoms only reached high abundances in late winter), we chose to focus solely on epiphytic macroalgae for the present study. Species identifications were made to the lowest taxonomic level possible (usually to species). We then spun all epibiont-free host samples 15 times in a salad spinner to remove excess water and measured their respective wet masses. Due to their small size, we blotted the epiphytes and then measured their wet masses.

Algal epiphyte abundance was standardized to both primary (rock) and secondary (host algae) substrate.
Epiphyte biomass was calculated per host sample to reflect the total biomass of epiphytes supported by each host per square centimeter of primary substrate. In addition, the density of epiphyte biomass per gram host was calculated to measure the amount of epiphytes supported per gram of secondary substrate. Epiphyte diversity was determined for each month using the Shannon-Weiner index. Epiphyte richness and diversity were both calculated per host sample. Epiphyte biomass, density, diversity, and richness data were first analyzed using a factorial nested analysis of variance (ANOVA) design, in which host species were nested within host type (native or invasive) and host type was crossed with month (MacANOVA V. 5.05). Host species and month were considered random factors, while host type was considered a fixed factor. Transect, a random factor, was also nested within date to account for variation between transects. Because we found a non-significant effect of host type and transect on all response variables (see Table 2), we re-ran our analyses for biomass, density, and richness using 2-way mixed-model ANOVAs (MacANOVA V. 5.05) to test for interspecific and temporal variability; diversity showed a significant transect(month) $\times$ host species (host type) interaction, so we used the full nestedmodel analysis. Host species was considered a fixed factor, while month was a random factor. Significant p-values were adjusted for multiple comparisons (Rice 1990), and Tukey honestly significance difference (HSD) post hoc comparisons were performed on significant univariate factors to identify sources of variation. All analyses were performed on untransformed data because transformations did not improve variance heterogeneity or normality and ANOVAs are robust to departures from these assumptions when replication is high (Underwood 1997).

Differences in epiphytic algal assemblages among host samples and months were tested using a permutational analysis of variance (PERMANOVA) (Anderson 2001), on a Bray-Curtis dissimilarity matrix of untransformed epiphyte densities. This non-parametric multivariate technique allows calculation of an interaction term in 2-factor analyses with mixed and random factors. Pair-wise a posteriori comparisons were performed on significant factors (Anderson 2001). Similarity percentage (SIMPER) analysis (Clarke 1993) was used to determine which epiphyte species were responsible for within-host similarity during the entire sampling period (Primer V. 6.0, Primer-E); species that contributed to at least $5 \%$ of host similarity were considered characteristic species of that assemblage.

Mimic experiments of secondary substrate characteristics. We investigated the importance of secondary substrate relief and host height for recruitment and growth of epiphytic algal species by conducting mimic 
experiments that manipulated these characteristics. Two different types of substrate were used: high-relief woven $0.6 \mathrm{~cm}$ diameter green rope and low-relief $1 \mathrm{~cm}$ wide green plastic strips (cut from plastic folders; Bouma et al. 2005). We cut each substrate to lengths of either 7 or $28 \mathrm{~cm}$ and attached 3 vertical 'branches' (same substrate and length) to a stainless steel washer. The mimics were attached to rock in the low intertidal at Brenton Point using marine epoxy (ZSPAR splash zone compound) during July 2006. Mimics were placed in a randomized complete block design, with 1 mimic of each type per block (long rope, short rope, long plastic, short plastic) and 20 blocks. After $8 \mathrm{wk}$, remaining mimics were removed and taken back to the laboratory (11 mimics were lost), where 1 branch was randomly selected for processing. After taking initial photographs, we removed, identified, blotted dry, and measured the wet mass of all epiphyte species. We calculated epiphyte biomass, density, richness, and Shannon-Weiner diversity for each mimic (all per sample except density, which was calculated as grams per square centimeter of substrate). Only 12 complete blocks remained when removed from the field, and these data were analyzed using a 2-way ANOVA with substrate and height as fixed factors (JMP V. 7.0). Block was analyzed as a third, non-interactive variable to account for random environmental variability.

Surveys of Lacuna vincta abundance. In order to examine the seasonal dynamics of $L$. vincta (hereafter referred to as Lacuna) at our sites, we recorded the number of snails on each algal sample collected from September 2005 to September 2006 at Brenton Point. We also counted the number of egg cases found on each host species from June 2005 to September 2006. Lacuna abundance was standardized to total grams of algae (host + epiphyte wet mass), because snails were found on both types. In contrast, egg cases were found solely on host algae and, thus, were standardized to grams of host mass. We analyzed data using 2-way mixed-model ANOVAs, with host species as a fixed factor and month as a random factor (MacANOVA V. 5.05).

We also examined the potential influences of the 2 most abundant epiphytes in our system, Ceramium virgatum and Neosiphonia harveyi (hereafter referred to by genus alone), on Lacuna recruitment. We used linear regressions (JMP V. 7.0), with the number of snails and Ceramium or Neosiphonia wet mass (g) from July and August 2006 (the months with peak juvenile Lacuna) to determine if there was a positive correlation between the number of snails and epiphyte biomass. Log transformations were done, if appropriate, prior to analysis.

Lacuna feeding preferences. In order to determine whether Lacuna preferred consuming one epiphytic algal species over another, we conducted paired- choice feeding assays between Ceramium virgatum and Neosiphonia harveyi. We haphazardly collected snails in the field and placed 3 Lacuna into each flowthrough plastic container (0.5 l) submerged in outdoor flowing seawater tables at the University of Rhode Island's Graduate School of Oceanography. Preliminary studies (E. Jones unpubl. data) showed that 3 was the minimum number of snails needed for measurable algal consumption before algae started to degrade (approximately $3 \mathrm{~d}$ ). We placed $0.05 \mathrm{~g}$ (salad-spun 15 times and blotted twice) of both Ceramium and Neosiphonia into each container $(\mathrm{n}=20)$. After $3 \mathrm{~d}$, the epiphyte samples were removed, re-spun, blotted dry, and then the wet mass was measured. As a control for changes in algal mass unrelated to herbivory, we simultaneously submerged identical containers with the algae being tested $(\mathrm{n}=20)$, but with no snails. Consumption was calculated as $\left(H_{\mathrm{i}} \times C_{\mathrm{f}} \times C_{\mathrm{i}}^{-1}\right)-H_{\mathrm{f}}$, where $H_{\mathrm{i}}$ and $H_{\mathrm{f}}$ were the initial and final mass of the algae and $C_{\mathrm{f}}$ and $C_{\mathrm{i}}$ were the final and initial mass of the controls (Stachowicz \& Hay 1999). Choice assays were analyzed using a paired $t$-test (JMP V. 7.0).

\section{RESULTS}

At Brenton Point, all 4 host species supported a large suite of epiphytic algal species, with variation in $\beta$-diversity. We found a total of 50 epiphyte species from June 2005 to September 2006 (Table 1; Ectocarpus fasciculatus and E. siliculosus are combined due to incomplete separation), with the largest number occurring on Codium (46), followed by Chondrus (35), Fucus (28), and Grateloupia (24). Sixteen epiphyte species were found on all 4 host species, and 9 were found only on a single host species (6 on Codium, 2 on Chondrus, and 1 on Fucus). With the exception of Elachista fucicola on Fucus, all of the species unique to a single host were found in a very small number of samples $(<4)$.

\section{Temporal and host variation in algal epiphyte abundance, richness, and diversity}

We found no significant effect of host type (native/ invasive) on algal epiphyte biomass, density, richness, or diversity (Table $2 ; \mathrm{p}>0.637$ ). There were highly significant effects of individual host species on all epiphyte characteristics, as described below.

We found significant differences in biomass among host species (Table 3, Fig. 1a; p < 0.0001). Mean epiphyte biomass on Codium was $4.36 \mathrm{~g} \mathrm{~cm}^{-2}$ of primary substrate, which was 2 to 10 times greater than on all other host species (Tukey HSD, p < 0.05). Fucus supported $1.91 \mathrm{~g}$ epiphytes $\mathrm{cm}^{-2}$ of primary substrate, over 
Table 1. Epiphytic algal species found at Brenton Point on each host species ( $\geq 1$ time) for each season. Sp: spring; S: summer; F: fall; W: winter; All: all seasons; - : not found ${ }^{*}$ : known invasives

\begin{tabular}{|c|c|c|c|c|}
\hline \multirow{2}{*}{ Epiphyte species } & \multirow[b]{2}{*}{ Codium fragile } & \multicolumn{2}{|c|}{ - Host species } & \multirow[b]{2}{*}{ Grateloupia turuturu } \\
\hline & & Chondrus crispus & Fucus vesiculosus & \\
\hline \multicolumn{5}{|l|}{ Rhodophyta } \\
\hline Antithamnion cruciatum & $\mathrm{S}$ & $\mathrm{S}$ & - & - \\
\hline Antithamnion pectinatum* ${ }^{*}$ & All & All & $\mathrm{S}, \mathrm{F}$ & $\mathrm{S}, \mathrm{F}$ \\
\hline Audouinella spp. & W, Sp & $\mathrm{S}$ & $\mathrm{W}, \mathrm{S}$ & - \\
\hline Bonnemaisonia hamifera* & Sp & - & - & - \\
\hline Callithamnion tetragonum & All & $\mathrm{Sp}$ & $\mathrm{W}, \mathrm{Sp}, \mathrm{S}$ & $\mathrm{S}, \mathrm{F}$ \\
\hline Ceramium deslongchampsii & $\mathrm{S}, \mathrm{F}$ & S & - & $\mathrm{S}$ \\
\hline Ceramium virgatum & All & $\mathrm{SP}, \mathrm{S}, \mathrm{F}$ & All & All \\
\hline Champia parvula & $\mathrm{S}$ & $\mathrm{Sp}, \mathrm{S}, \mathrm{F}$ & $\mathrm{S}, \mathrm{F}$ & $\mathrm{S}, \mathrm{F}$ \\
\hline Chondria baileyana & $\mathrm{S}$ & $\mathrm{S}$ & - & $\mathrm{S}$ \\
\hline Chondria capillaris & $\mathrm{S}$ & $\mathrm{S}$ & - & - \\
\hline Chondrus crispus & $\mathrm{Sp}, \mathrm{S}$ & - & - & - \\
\hline Corallina officinalis & W, Sp, S & $\mathrm{Sp}, \mathrm{S}$ & - & $\mathrm{Sp}, \mathrm{S}$ \\
\hline Cystoclonium purpureum & $\mathrm{Sp}, \mathrm{S}$ & All & $\mathrm{Sp}, \mathrm{S}$ & $\mathrm{Sp}, \mathrm{S}$ \\
\hline Gelidium pusillum & $\mathrm{W}, \mathrm{Sp}, \mathrm{S}$ & $\mathrm{Sp}, \mathrm{S}$ & - & $\mathrm{W}, \mathrm{S}$ \\
\hline Neosiphonia harveyi* & All & All & All & All \\
\hline Polysiphonia fucoides & $\mathrm{Sp}, \mathrm{S}$ & $\mathrm{Sp}$ & - & - \\
\hline Polysiphonia nigra & $\mathrm{S}$ & $\mathrm{Sp}$ & - & - \\
\hline Polysiphonia stricta & $\mathrm{Sp}, \mathrm{S}$ & $\mathrm{S}$ & - & $\mathrm{F}$ \\
\hline Porphyra umbilicalis & $\mathrm{W}, \mathrm{Sp}$ & $\mathrm{W}, \mathrm{Sp}$ & $\mathrm{W}, \mathrm{Sp}, \mathrm{S}$ & W, Sp \\
\hline Spermothamnion repens & $\mathrm{W}, \mathrm{Sp}, \mathrm{S}$ & $\mathrm{Sp}, \mathrm{S}, \mathrm{F}$ & $\mathrm{S}$ & $\mathrm{W}, \mathrm{S}$ \\
\hline Vertebrata lanosa & $\mathrm{Sp}$ & - & - & - \\
\hline Red crust (non-coralline) & $\mathrm{W}$ & - & $\mathrm{Sp}$ & - \\
\hline Unknown red blade & $\mathrm{Sp}$ & $\mathrm{Sp}$ & - & $\mathrm{Sp}$ \\
\hline \multicolumn{5}{|l|}{ Chlorophyta } \\
\hline Chaetomorpha linum & S, F & All & $\mathrm{S}$ & $\mathrm{S}$ \\
\hline Chaetomorpha melagonium & $\mathrm{W}, \mathrm{Sp}, \mathrm{S}$ & $\mathrm{W}, \mathrm{Sp}, \mathrm{S}$ & $\mathrm{S}, \mathrm{F}$ & $\mathrm{W}, \mathrm{Sp}, \mathrm{S}$ \\
\hline Cladophora albida & $\mathrm{S}$ & $\mathrm{Sp}, \mathrm{S}$ & $\mathrm{S}, \mathrm{F}$ & $\mathrm{Sp}$ \\
\hline Cladophora sericea & $\mathrm{S}$ & $\mathrm{Sp}, \mathrm{S}$ & $\mathrm{Sp}, \mathrm{S}$ & - \\
\hline Codium fragile* & - & $\mathrm{Sp}$ & - & - \\
\hline Ulothrix flacca & $\mathrm{Sp}$ & - & W & - \\
\hline Ulva compressa & W, Sp, S & - & W, Sp, S & - \\
\hline Ulva intestinalis & $\mathrm{Sp}, \mathrm{S}$ & $\mathrm{Sp}, \mathrm{S}$ & $\mathrm{Sp}, \mathrm{S}$ & - \\
\hline Ulva lactuca & $\mathrm{W}, \mathrm{Sp}, \mathrm{S}$ & $\mathrm{W}, \mathrm{Sp}, \mathrm{S}$ & All & W, Sp \\
\hline Ulva linza & W & $\mathrm{Sp}$ & - & W \\
\hline Unknown filamentous green & $\mathrm{W}$ & - & - & - \\
\hline \multicolumn{5}{|l|}{ Phaeophyceae } \\
\hline Aspercoccus fistulosus & $\mathrm{Sp}$ & $\mathrm{S}$ & $\mathrm{Sp}$ & - \\
\hline Chordaria flagelliformis & $\mathrm{Sp}, \mathrm{S}$ & $\mathrm{Sp}, \mathrm{S}$ & $\mathrm{Sp}, \mathrm{S}$ & $\mathrm{Sp}$ \\
\hline Ectocarpus spp. & W, Sp, S & $\mathrm{Sp}, \mathrm{S}$ & $\mathrm{Sp}, \mathrm{S}$ & $\mathrm{Sp}$ \\
\hline Elachista chondrii & - & $\mathrm{W}, \mathrm{Sp}, \mathrm{S}$ & - & - \\
\hline Elachista fucicola & - & - & All & - \\
\hline Elachista sp. & $\mathrm{Sp}, \mathrm{S}$ & - & - & $\mathrm{Sp}$ \\
\hline Leathesia difformis & $\mathrm{Sp}, \mathrm{S}$ & $\mathrm{Sp}, \mathrm{S}$ & $\mathrm{Sp}, \mathrm{S}$ & $\mathrm{Sp}$ \\
\hline Petalonia fascia & $\mathrm{Sp}$ & - & - & - \\
\hline Punctaria latifolia & $\mathrm{W}, \mathrm{Sp}, \mathrm{S}$ & $\mathrm{S}$ & W, Sp & $\mathrm{Sp}$ \\
\hline Punctaria plantaginea & $\mathrm{Sp}$ & - & $\mathrm{Sp}$ & - \\
\hline Scytosiphon lomentaria & W, Sp & - & W, Sp & - \\
\hline Sphacelaria cirrosa & All & $\mathrm{Sp}, \mathrm{S}, \mathrm{F}$ & $\mathrm{Sp}, \mathrm{S}, \mathrm{F}$ & - \\
\hline Spongonema tomentosus & $\mathrm{Sp}, \mathrm{S}$ & $\mathrm{S}$ & W, Sp, S & - \\
\hline Unknown brown knob & $\mathrm{S}$ & - & - & - \\
\hline Unknown branched brown & Sp & - & - & - \\
\hline Total & 46 & 35 & 28 & 24 \\
\hline
\end{tabular}

3 times greater than on Chondrus $\left(0.643 \mathrm{~g} \mathrm{~cm}^{-2}\right)$ and Grateloupia (0.454 $\mathrm{g} \mathrm{cm}^{-2}$ ) (Tukey HSD, p < 0.05). All hosts showed increases in epiphyte biomass during the spring and/or summer and declines during the fall and winter, with the duration and magnitude of epiphyte biomass change being small on Fucus and Grateloupia and much greater on Chondrus and Codium (Fig. 1a). Although the effect of month was marginally insignificant (Table $3 ; \mathrm{p}=0.0648$ ), there was a significant month $\times$ host species interaction (Table $3 ; \mathrm{p}<0.0001$ ). 
Table 2. Results of nested ANOVAs testing for differences in epiphyte biomass, density, richness, and diversity among host type and month, with species nested within host; $\mathrm{p}$-values that remained significant after adjusting for multiple comparisons are in bold

\begin{tabular}{|c|c|c|c|c|}
\hline Source & df & MS & $F$ & $\mathrm{p}$ \\
\hline \multicolumn{5}{|l|}{ Biomass } \\
\hline Month & 11 & 44.2 & 3.23 & 0.032 \\
\hline Host type & 1 & 154 & 0.304 & 0.6370 \\
\hline Host species (Host type) & 2 & 506 & 53.9 & $<0.0001$ \\
\hline Month $\times$ Host type & 11 & 13.7 & 0.511 & 0.875 \\
\hline Transect (Month) & 12 & 8.56 & 1.25 & 0.352 \\
\hline $\begin{array}{l}\text { Month } \times \text { Host species } \\
\text { (Host type) }\end{array}$ & 22 & 26.7 & 2.85 & $<0.0001$ \\
\hline $\begin{array}{l}\text { Transect }(\text { Month) } \times \\
\text { Host type }\end{array}$ & 12 & 6.85 & 0.733 & 0.707 \\
\hline $\begin{array}{l}\text { Transect (Month) } \times \\
\text { Host species (Host type) }\end{array}$ & 24 & 9.34 & 0.996 & 0.471 \\
\hline Residual & 384 & 9.39 & & \\
\hline \multicolumn{5}{|l|}{ Density } \\
\hline Month & 11 & 0.780 & 4.85 & 0.0072 \\
\hline Host type & 1 & 0.403 & 0.207 & 0.694 \\
\hline Host species (Host type) & 2 & 1.95 & 4.80 & 0.0087 \\
\hline Month $\times$ Host type & 11 & 0.161 & 0.432 & 0.925 \\
\hline Transect (Month) & 12 & 0.293 & 0.780 & 0.663 \\
\hline $\begin{array}{l}\text { Month } \times \text { Host species } \\
\text { (Host type) }\end{array}$ & 22 & 0.372 & 0.919 & 0.570 \\
\hline $\begin{array}{l}\text { Transect }(\text { Month }) \times \\
\text { Host type }\end{array}$ & 12 & 0.375 & 1.18 & 0.352 \\
\hline $\begin{array}{l}\text { Transect (Month) } \times \\
\text { Host species (Host type) }\end{array}$ & 24 & 0.319 & 0.786 & 0.755 \\
\hline Residual & 384 & 0.405 & & \\
\hline \multicolumn{5}{|l|}{ Richness } \\
\hline Month & 11 & 41.1 & 1.92 & 0.147 \\
\hline Host type & 1 & 12.0 & 0.336 & 0.872 \\
\hline Host species (Host type) & 2 & 358 & 82.7 & $<0.0001$ \\
\hline Month $\times$ Host type & 11 & 21.4 & 1.05 & 0.443 \\
\hline Transect (Month) & 12 & 8.25 & 2.29 & 0.0833 \\
\hline $\begin{array}{l}\text { Month } \times \text { Host species } \\
\text { (Host type) }\end{array}$ & 22 & 20.4 & 4.72 & $<0.0001$ \\
\hline $\begin{array}{l}\text { Transect }(\text { Month }) \times \\
\text { Host type }\end{array}$ & 12 & 3.61 & 0.732 & 0.708 \\
\hline $\begin{array}{l}\text { Transect (Month) } \times \\
\text { Host species (Host type) }\end{array}$ & 24 & 4.93 & 1.14 & 0.297 \\
\hline Residual & 384 & 4.33 & & \\
\hline \multicolumn{5}{|l|}{ Diversity } \\
\hline Month & 11 & 0.651 & 2.18 & 0.106 \\
\hline Host type & 1 & 0.104 & 0.0159 & 0.911 \\
\hline Host species (Host type) & 2 & 6.57 & 56.7 & $<0.0001$ \\
\hline Month $\times$ Host type & 11 & 0.299 & 0.926 & 0.534 \\
\hline Transect (Month) & 12 & 0.133 & 1.10 & 0.434 \\
\hline $\begin{array}{l}\text { Month } \times \text { Host species } \\
\text { (Host type) }\end{array}$ & 22 & 0.323 & 2.78 & $<0.0001$ \\
\hline $\begin{array}{l}\text { Transect }(\text { Month }) \times \\
\text { Host type }\end{array}$ & 12 & 0.121 & 0.550 & 0.860 \\
\hline $\begin{array}{l}\text { Transect (Month) } \times \\
\text { Host species (Host type) }\end{array}$ & 24 & 0.220 & 1.89 & 0.0073 \\
\hline Residual & 384 & 0.116 & & \\
\hline
\end{tabular}

Table 3. Results of 2-way ANOVAs testing for differences in epiphyte biomass, density, and richness among hosts and months; $\mathrm{p}$-values that remained significant after adjusting for multiple comparisons are in bold

\begin{tabular}{|lrccr|}
\hline Source & df & MS & $F$ & $\mathrm{p}$ \\
\hline Biomass & & & & \\
$\quad$ Month & 11 & 44.2 & 1.97 & 0.0648 \\
Host species & 3 & 389 & 41.8 & $<\mathbf{0 . 0 0 0 1}$ \\
Month $\times$ Host species & 33 & 22.4 & 2.41 & $\mathbf{< . 0 0 0 1}$ \\
Residual & 432 & 9.29 & & \\
& & & & \\
Density & & & & \\
$\quad$ Month & 11 & 0.780 & 2.58 & 0.0173 \\
Host species & 3 & 1.43 & 3.61 & $\mathbf{0 . 0 1 3 4}$ \\
Month $\times$ Host species & 33 & 0.302 & 0.761 & 0.829 \\
Residual & 432 & 0.396 & & \\
& & & & \\
Richness & & & & \\
$\quad$ Month & 11 & 41.1 & 1.98 & 0.0639 \\
Host species & 3 & 243 & 54.5 & $<\mathbf{0 . 0 0 0 1}$ \\
Month $\times$ Host species & 33 & 20.7 & 4.66 & $<\mathbf{0 . 0 0 0 1}$ \\
Residual & 432 & 4.45 & & \\
\hline
\end{tabular}

Mean density was greatest on Chondrus and Codium (0.327 and $0.240 \mathrm{~g}$ epiphytes $\mathrm{g}^{-1}$ host, respectively) and less on Fucus (0.120 g epiphytes $\mathrm{g}^{-1}$ host) and Grateloupia (0.0916 g epiphytes $\mathrm{g}^{-1}$ host; Fig. 1b, Table $3 ; \mathrm{p}=0.0134$ among hosts), with the density on Chondrus being significantly greater than on Grateloupia (Tukey HSD, p < 0.05). Over the sampling period, density was greatest in early- to mid-summer ( 0.273 to $0.560 \mathrm{~g}$ epiphytes $\mathrm{g}^{-1}$ host) and lowest in the fall and late winter (0.0586 to $0.0994 \mathrm{~g}$ epiphytes $\mathrm{g}^{-1}$ host; Table 3; $\mathrm{p}=0.0173$ ), although the effect of month was not significant after adjusting for multiple comparisons. There was no significant month $\times$ host species interaction (Table 3; p = 0.829).

Fucus and Grateloupia had generally low algal epiphyte richness throughout the year, while Chondrus had seasonally greater epiphyte richness during the summer months, and Codium maintained a high richness of epiphytes over time (Fig. 1c, Table 3 ; p < 0.0001 for both host species and the interaction). Codium supported significantly more species per sample (mean = 5.40 species) than did Chondrus (4.25 species), and both supported more species than did Fucus (2.78 species) and Grateloupia (2.27 species; Tukey HSD, p < 0.05). These patterns were similar for diversity (Fig. 1d); host species nested within host type was highly significant (Table 2; p <0.0001), with Chondrus and Codium having significantly greater diversity (0.547 and 0.573, respectively) compared to Fucus (0.277) and Grateloupia (0.191; Tukey HSD, p < 0.05). There was also a significant host month $\times$ species (host type) interaction. When all sources of variation were 

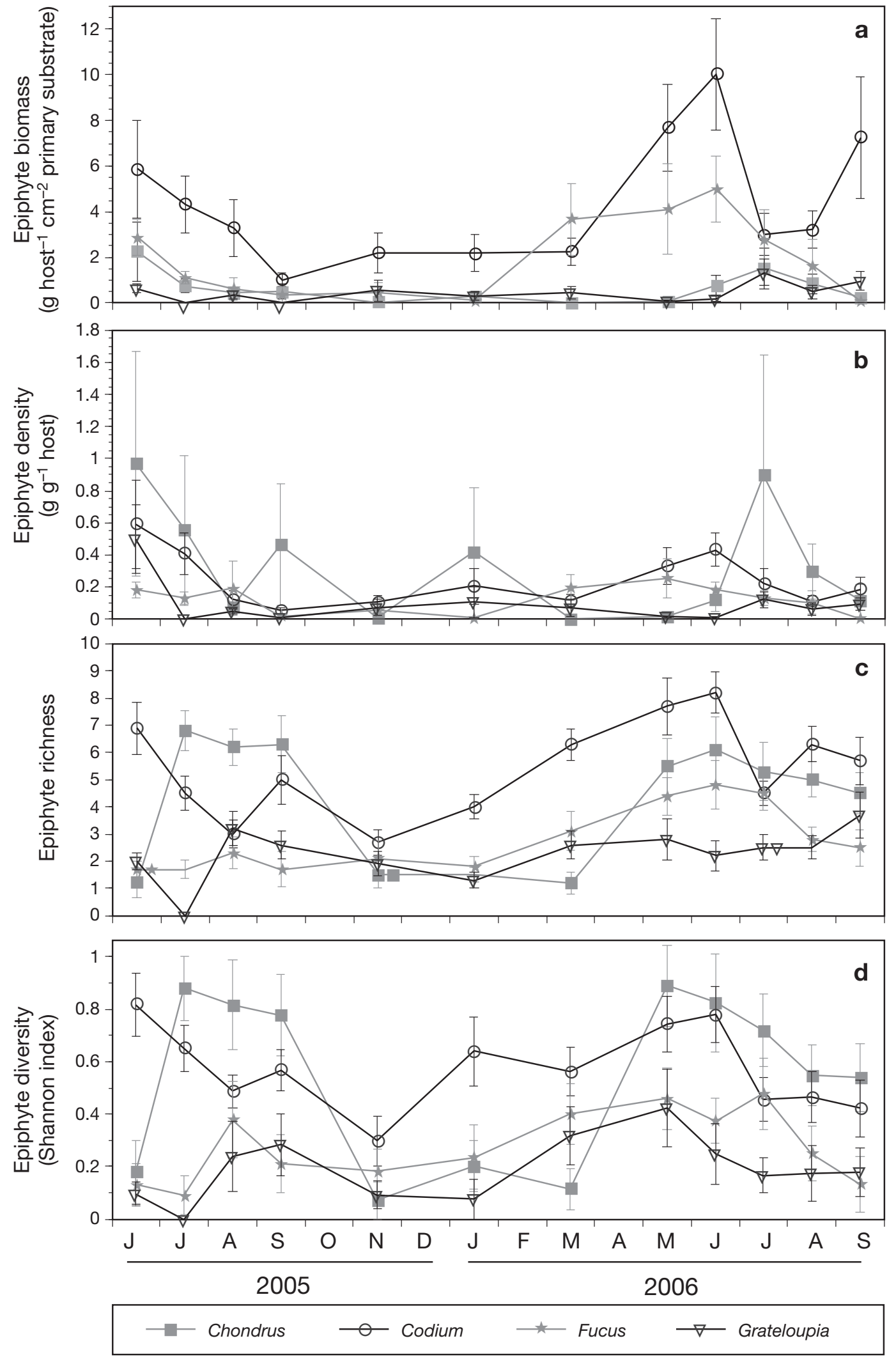

Fig. 1. Temporal variation in epiphyte (a) biomass, (b) density, (c) richness, and (d) diversity among host species (means \pm SE), Chondrus crispus, Codium fragile, Fucus vesiculosus, Grateloupia turuturu 
taken into account, there was also a significant transect (month) $\times$ host species (host type) interaction (Table 2; $\mathrm{p}=0.0073$ ); this appears to be driven by 2 months that had a high epiphyte diversity on certain transects.

\section{Epiphyte community analysis}

We found large and significant differences in algal epiphyte community structure among hosts and months, as well as a significant interaction; thus, differences in epiphyte community structure were found among hosts depending on month (PERMANOVA, $\mathrm{p}=$ 0.0001 for all; Table 4). Pair-wise a posteriori comparisons of hosts for these assemblages showed that between-host dissimilarity for all months ranged from $46 \%$ (Chondrus/Grateloupia and Codium/Grateloupia) to $61 \%$ (Fucus/Codium) ( $\mathrm{p}=0.0002$ to 0.0068 ). Pairwise a posteriori comparisons of months showed that June 2006 and January 2006 had the greatest dissimilarity (95\%), while most adjacent months (i.e. July/ August) were not significantly different from each other ( $p=0.0002$ to 0.440 ). Individual a posteriori comparisons of host by date yielded a more complex pattern. Comparisons of epiphyte assemblages were significantly dissimilar ( $p=0.0002$ to 0.0242 ), with the following exceptions: Codium and Grateloupia during June 2005, November 2005, January 2006, March 2006, July 2006, and September 2006 ( $\mathrm{p}=0.0556$ to 0.378); Chondrus and Grateloupia during August 2005, July 2006, and August 2006 ( $p=0.0830$ to 0.179); Fucus and Chondrus and Fucus and Grateloupia during November 2005 ( $\mathrm{p}=0.303$ and 0.432); and Chondrus and Codium during May 2006 ( $\mathrm{p}=0.131)$.

Characteristic epiphytic algal species for each host species over the entire sampling period were determined using SIMPER analysis on epiphyte density data (Table 5). Chondrus had the largest suite of species (5), followed by Codium (3), Fucus (2), and Grateloupia (1). Many of the species were seasonally present: on Chondrus, Leathesia difformis was only found from May to July, Spermothamnion repens and Ceramium virgatum were found from May to November, and Corallina

Table 4. Results of a permutational ANOVA testing for dissimilarity of epiphyte assemblages among hosts and months. Significant $p$-values are in bold

\begin{tabular}{|lrrcc|}
\hline Source & df & MS & $F$ & $p$ \\
\hline Month & 11 & 10616 & 3.18 & $\mathbf{0 . 0 0 0 1}$ \\
Host & 3 & 77952 & 9.27 & $\mathbf{0 . 0 0 0 1}$ \\
Month $\times$ Host & 33 & 8408 & 2.52 & $\mathbf{0 . 0 0 0 1}$ \\
Residual & 432 & 3342 & & \\
\hline
\end{tabular}

Table 5. Results of similarity percentage (SIMPER) analysis showing the percent contribution of epiphyte species to community structure. Data are for species that contributed at least $2 \%$ to within-host similarity; characteristic species ( $\geq 5 \%$ ) for each host are in bold

\begin{tabular}{|llr|}
\hline \multirow{2}{*}{ Host } & Species & $\%$ \\
\hline \multirow{6}{*}{ Fucus vesiculosus } & Elachista fucicola & $\mathbf{8 7 . 2}$ \\
& Porphyra umbilicalis & $\mathbf{6 . 8}$ \\
& Neosiphonia harveyi & 2.6 \\
& Ceramium virgatum & 2.0 \\
& Neosiphonia harveyi & $\mathbf{3 0 . 4}$ \\
& Antithamnion pectinatum & $\mathbf{2 1 . 5}$ \\
& Leathesia difformis & $\mathbf{1 2 . 5}$ \\
& Spermothamnion repens & $\mathbf{1 1 . 0}$ \\
& Corallina officinalis & $\mathbf{7 . 0}$ \\
& Ceramium virgatum & 4.6 \\
& Ectocarpus fasciculatus & 4.4 \\
& Ceramium deslongchampsii & 2.8 \\
& Neosiphonia harveyi & $\mathbf{5 2 . 2}$ \\
& Ceramium virgatum & $\mathbf{3 9 . 1}$ \\
& Ectocarpus spp. & $\mathbf{7 . 6}$ \\
Grateloupia turuturu & Neosiphonia harveyi & $\mathbf{9 3 . 9}$ \\
& Ectocarpus spp. & 2.8 \\
& Ceramium virgatum & 2.2 \\
& & \\
& &
\end{tabular}

officinalis was found from May to September, while Porphyra umbilicalis was only found on Fucus from January to July, and Ectocarpus spp. were only found on Codium from March to July (Table 1).

\section{Mimics of host structure}

Structural mimics of secondary substrate showed that the high-relief rope supported 115 times greater algal epiphyte biomass than the low-relief plastic (1.92 vs. $0.0167 \mathrm{~g}$ ), 2.5 times as many species (6.38 vs. 2.54), and a mean diversity of 0.587 vs. 0.230 . This effect of substrate relief was significant for biomass, density, richness, and diversity (Fig. 2, Table 6; p < 0.007). However, mimics showed no effect of height on epiphyte richness or diversity (Fig. 2, Table 6; richness, $\mathrm{p}=1.00$; diversity, $\mathrm{p}=0.685$ ). Although taller mimics supported a larger epiphyte biomass per branch $(1.53 \mathrm{~g})$ than shorter ones $\left(0.401 \mathrm{~g}_{i} \mathrm{p}=0.0037\right)$, the density of epiphytes per square centimeter of substrate did not significantly differ $(p=0.927)$. There was a significant interaction between height and substrate on biomass ( $p=0.0046$ ), with the long rope supporting a greater total biomass than the other mimics (Tukey HSD, $\mathrm{p}<0.05)$. A total of 24 species recruited to the mimics during the summer; 21 of these were present on the rope and 13 on the plastic. Eighteen of these epiphytes were also found on host algae. 

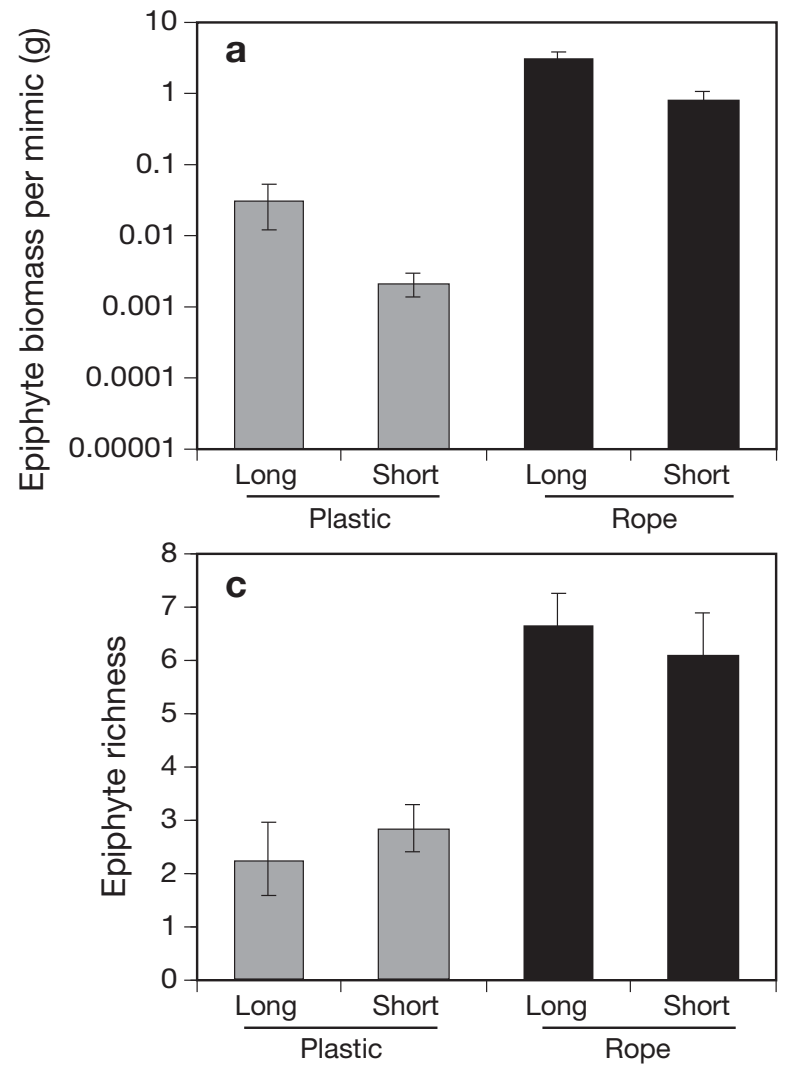
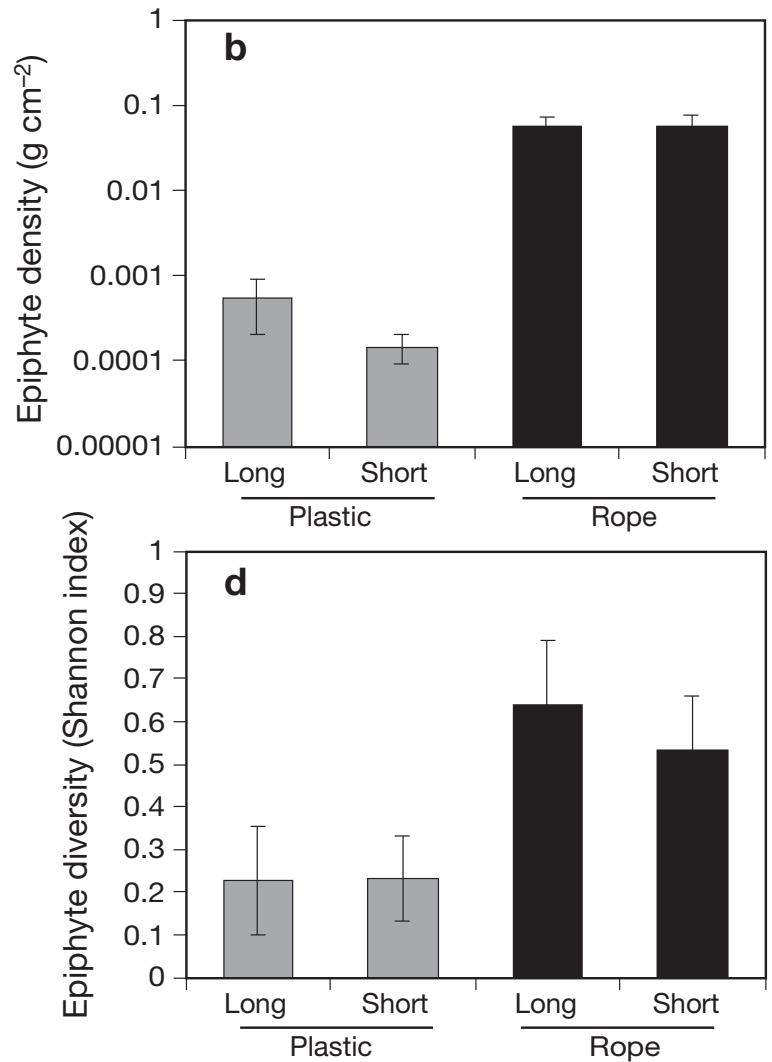

Fig. 2. Variation in epiphyte (a) biomass, (b) density, (c) richness, and (d) diversity among mimic substrates (means \pm SE)

Table 6. Results of 2-way ANOVAs testing for differences in epiphyte biomass, density, richness, and diversity among mimic height and substrate relief. Significant p-values are in bold

\begin{tabular}{|lcccc|}
\hline Source & df & MS & $F$ & $\mathrm{p}$ \\
\hline Biomass & & & & \\
$\quad$ Substrate & 1 & 43.3 & 27.5 & $<\mathbf{0 . 0 0 0 1}$ \\
Height & 1 & 15.4 & 9.76 & $\mathbf{0 . 0 0 3 7}$ \\
Substrate $\times$ Height & 1 & 14.6 & 9.26 & $\mathbf{0 . 0 0 4 6}$ \\
Block & 11 & 2.30 & 1.46 & 0.194 \\
Residual & 33 & 1.57 & & \\
Density & & & & \\
Substrate & 1 & 0.0403 & 36.6 & $<\mathbf{0 . 0 0 0 1}$ \\
Height & 1 & $9.48 \times 10^{-6}$ & 0.0086 & 0.927 \\
Substrate $\times$ Height & 1 & $2.03 \times 10^{-5}$ & 0.0184 & 0.893 \\
Block & 11 & 0.00210 & 1.91 & 0.0750 \\
Residual & 33 & 0.0011 & & \\
Richness & & & & \\
$\quad$ Substrate & 1 & 176 & 31.2 & $<\mathbf{0 . 0 0 0 1}$ \\
Height & 1 & 0 & 0 & 1.00 \\
Substrate $\times$ Height & 1 & 4.08 & 0.722 & 0.402 \\
Block & 11 & 2.27 & 0.401 & 0.946 \\
$\quad$ Residual & 33 & 5.65 & & \\
Diversity & & & & \\
$\quad$ Substrate & 1 & 1.53 & 8.28 & $\mathbf{0 . 0 0 7 0}$ \\
Height & 1 & 0.031 & 0.167 & 0.685 \\
Substrate $\times$ Height & 1 & 0.037 & 0.201 & 0.657 \\
Block & 11 & 0.202 & 1.09 & 0.397 \\
Residual & 33 & 0.185 & & \\
\hline & & & & \\
\hline
\end{tabular}

\section{Lacuna surveys and feeding preferences}

Lacuna egg case abundance (no. $\mathrm{g}^{-1}$ host) peaked in the winter and dropped off to zero during the spring and summer (Fig. 3), with most egg cases being found on Chondrus $\left(F_{11,432}=1.17, \mathrm{p}=0.347\right.$ for month; $F_{3,432}=$ $4.05, \mathrm{p}=0.0074$ for species; $F_{33,432}=2,83, \mathrm{p}<0.0001$ for the interaction). This was followed by a large spike in Lacuna abundance (no. $\mathrm{g}^{-1}$ total algae) during July and August, with densities reaching 16.5 snails $\mathrm{g}^{-1}$ algae $\left(F_{8,324}=5.56, \mathrm{p}=0.0005\right.$ for month, Tukey HSD, $\mathrm{p}<$ 0.05 for July and August). This seasonal trend was consistent for all hosts (with no significant host by month interaction; $F_{24,324}=1.35, \mathrm{p}=0.127$ ). Chondrus supported the greatest number of snails per gram algae $\left(F_{3,324}=7.32, \mathrm{p}=0.0001\right.$ among hosts, Tukey HSD, $\mathrm{p}<0.05$ for Chondrus). The number of Lacuna per host per square centimeter primary substrate did not vary among hosts $\left(F_{3,324}=1.76, \mathrm{p}=0.155\right)$.

The 2 most abundant algal epiphytes in this system were Neosiphonia harveyi and Ceramium virgatum, which were found in 65 and $53 \%$ of the samples collected, respectively, and contributed to $50-80 \%$ of the total epiphyte abundance during the summer months (when they were found on all host species). During Lacuna recruitment from June to August, Ceramium 


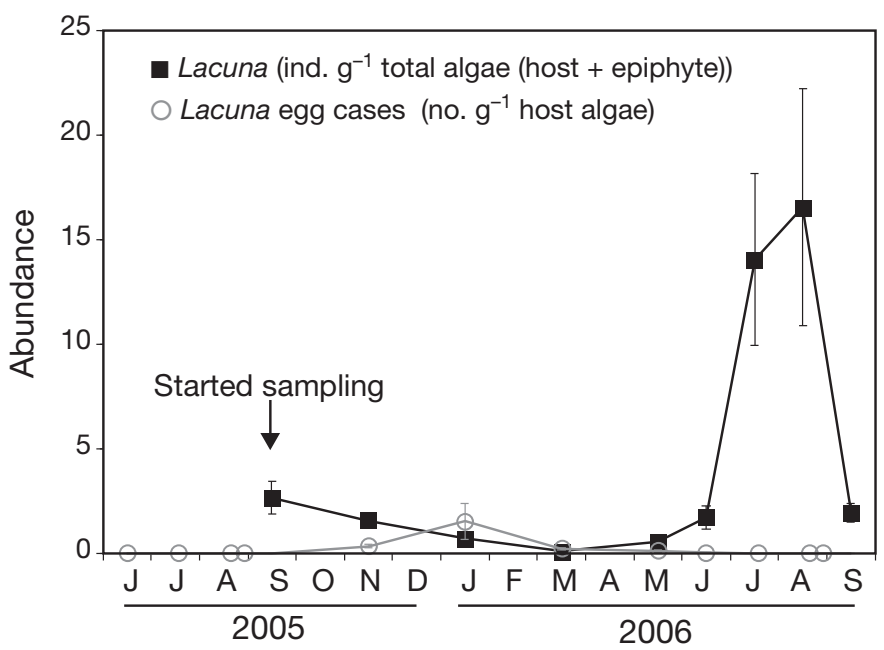

Fig. 3. Temporal variation in abundance of Lacuna individuals and number of egg cases at Brenton Point, RI. Monthly means $( \pm \mathrm{SE})$ are shown for simplicity; summer increases occurred on all hosts (2-way ANOVA, p $\leq 0.05$ for both month and host)

was in greatest abundance (both biomass and density) on Codium and Fucus, while Neosiphonia was in greatest abundance on Chondrus, Codium, and Grateloupia. Lacuna abundance during July and August was significantly correlated with Neosiphonia abundance (Fig. $4 ; \mathrm{r}^{2}=0.419, \mathrm{p}<0.0001$ ), while there was no significant relationship between Ceramium and Lacuna abundance $\left(\mathrm{r}^{2}=0.0132, \mathrm{p}=0.311\right)$.

When given a choice between these 2 most abundant epiphyte species in feeding trials, Lacuna consumed more than twice as much Ceramium as Neosiphonia tissue $(0.0074 \pm 0.0015$ (SE) vs. $0.0035 \pm$ $0.0012 \mathrm{~g}$, paired $t$-test, $t_{19}=-2.07, \mathrm{p}=0.05$ ).

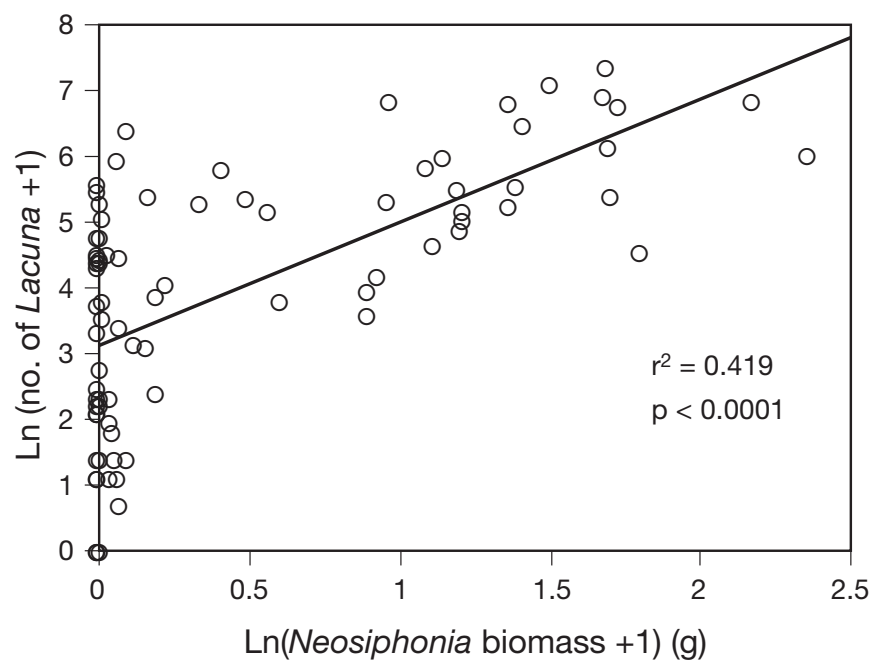

Fig. 4. Relationship between Lacuna abundance and Neosiphonia biomass on all host species. Analysis is by simple linear regression

\section{DISCUSSION}

\section{Temporal and habitat differences in epiphyte communities}

Ecological communities and species assemblages are often built upon the habitat that foundation species provide (Bruno \& Bertness 2001, Bruno et al. 2003). Numerous studies have highlighted how increased habitat heterogeneity of secondary substrate can positively affect smaller organisms (e.g. mussels: Lohse 1993; algae: Dean \& Connell 1987; coral: Idjadi \& Edmunds 2006; oysters: Grabowski et al. 2005; seagrass: Orth \& Heck 1980). In the present study, we found that individual host traits, rather than species origin, were important for supporting different assemblages of epiphytic algal species, with habitat-complexity and seasonality likely both being important factors in structuring these communities; similar results have been found for seaweed-epifaunal assemblages (Bates 2009).

During the summer months, recruitment of filamentous algal epiphytes was high, and structural complexity of the host appeared to be important for supporting high epiphyte richness and diversity. Chondrus, which has a complex tufted morphology, and Codium, which has a high surface relief and 3-dimensional branching, supported a 2 to 8 times greater density, a 2 times greater richness, and a 2 to 4 times greater diversity of epiphyte species than the more 2-dimensional Fucus (flat branches) and Grateloupia (flat blades) in the summer. Our mimic experiments supported these observations by suggesting that increased habitat complexity (e.g. surface relief), rather than simply host size, was important for differences in epiphyte density, richness, and diversity. In addition, like the long rope mimic with a combined greater height and surface relief, the taller and more complex Codium supported a far greater biomass than the other hosts. Similarly, during the summer, the more complex Sargassum muticum supported a greater epibiont richness compared to the less complex natives, Halidrys siliquosa (Wernberg et al. 2004) and Fucus vesiculosus (Buschbaum et al. 2006), in Europe. In addition, in Nova Scotia, the more complex invasive Codium fragile ssp. tomentosoides supported a greater epibiont richness and diversity compared to the less complex native, Laminaria spp. (Schmidt \& Scheibling 2006).

Plants, algae, and animals that create habitat often have mechanical and chemical means to prevent the settlement of other organisms (Filion-Myklebust \& Norton 1981, Moss 1982, Bakus et al. 1986, Davis et al. 1989). The native species used here can slough or shed tissue, thereby removing colonizing epibiotic organisms (Sieburth \& Tootle 1981, Russell \& Veltkamp 1984). During the summer months, rapid re- 
cruitment and subsequent growth by epiphytic algae (an average of from 0.058 to $0.098 \mathrm{~g} \mathrm{~cm}^{-2}$ over $8 \mathrm{wk}$ ) may have occurred faster than the sloughing capabilities of these hosts. In contrast, during the winter, slow epiphyte growth and recruitment rates may allow Chondrus and Fucus to effectively remove some epiphytes from their surfaces. Our data suggest that Codium may be important for supporting epiphytic algae during the winter months (Fig. 1); the high abundance of epiphytic algae during this time may be because Codium lacks a tissue structure for sloughing (Mathieson et al. 2003). Therefore, while structural mechanisms may explain the high richness and diversity of epiphytes found on Chondrus and Codium during the summer, other mechanisms, such as mechanical differences of hosts, may be more important during colder months. These seasonal changes may be important in other systems where foundation species, such as trees, salt marsh plants, and seagrasses, undergo seasonal morphological changes, especially when invasive species display different changes than native species.

Inhibition of epiphyte settlement by chemical properties may also explain why 2 of our host species had low algal epiphyte richness and diversity for the entire sampling period. Wikström \& Pavia (2004) found chemical inhibition of epiphytes by Fucus vesiculosus, and Grateloupia has been shown to exhibit antifouling properties (Hellio et al. 2004, Paul et al. 2006). This may explain the low number of species that settled directly on the hosts' tissues, especially Grateloupia, as most epiphyte species associated with Grateloupia grew on its dominant epiphyte, Neosiphonia harveyi. This epiphyte is also an invasive species of Asiatic origin (Choi et al. 2001), and it has also been found growing epiphytically on Grateloupia in other areas that Grateloupia has invaded (e.g. NW Spain; Barbàra \& Cremades 2004). Thus, even though an invasive species' morphology may be structurally suitable as habitat, chemical defense mechanisms may limit the usability of the host as habitat or food (Amade \& Lemee 1998).

These differences in richness and diversity are important, but perhaps most interesting is that these hosts support different assemblages of epiphytic algae, and differences among hosts depend on month (Table 4 , host by month interaction). While Codium supported the greatest number of epiphyte species, it was characterized by 3 species, 2 of which occurred in 117 of 120 samples (Ceramium virgatum and Neosiphonia harveyi). Although found on all hosts, these epiphytes were most abundant on Codium. Chondrus had the greatest number of characteristic species, many unique from the other hosts; these species were primarily found during the spring and summer. Al- though Fucus supported a much lower number of species, one of these species (Elachista fucicola) was an obligate epiphyte on fucoids (Villalard-Bohnsack 2003), while the other characteristic species (Porphyra umbilicalis) was dominant on Fucus during the winter. Thus, the presence of this host species is essential for these epiphyte species. Grateloupia was the only host that did not support unique epiphyte species; its 'assemblage' consisted solely of Neosiphonia. As a community, these host species play different roles for the numbers, identities, and biomasses of species that they support in both 2- and 3-dimensional space.

\section{Direct and indirect trophic-level interactions}

While our surveys focused on the effect of hosts on epiphytic algae, the reverse relationship is also important to consider. The interaction from epiphyte to host can be positive (Stewart 1982, Norton \& Benson 1983, Karez et al. 2000), neutral (Cattaneo 1983), or negative (Orth \& van Montfrans 1984, D'Antonio 1985, Williams $\&$ Seed 1992). Foundation species often depend on associated organisms, such as mesograzers, to decrease competition with, or overgrowth by, epibionts (Duffy 1990, Stachowicz \& Hay 1999, Stachowicz \& Whitlatch 2005).

Herbivores can synchronize recruitment events to coincide with increased abundances of food sources (e.g. Mattson 1980). In southern Rhode Island, Lacuna vincta began recruitment in late spring (May/June), reaching peak densities of juveniles in July and August (Fig. 3; similar to the results by Southgate 1982). The 2 most abundant algal epiphyte species, Neosiphonia harveyi and Ceramium virgatum, began recruitment in late June (E. Jones unpubl. data), with no change in average density on hosts in the field. It is possible that, through consumption, Lacuna may prevent epiphyte overgrowth on host species, thus, indirectly having a positive effect on the host algae (Stachowicz \& Whitlatch 2005). We did observe epiphyte grazing damage on colonized mimic substrates placed out in May and removed in August, following peak epiphyte recruitment in July (E. Jones unpubl. data). Due to the year-round growth of these epiphytes, however, it is difficult to determine the relationship between these snails and algae through survey data; the mechanisms underlying these epiphyte-snail interactions remain to be experimentally tested.

In addition to the potential indirect positive interaction from Lacuna on host algae, by supporting epiphytic algal growth, both native and invasive host algae may exhibit indirect positive effects on Lacuna (Fig. 5). Previous studies have found low abundances 


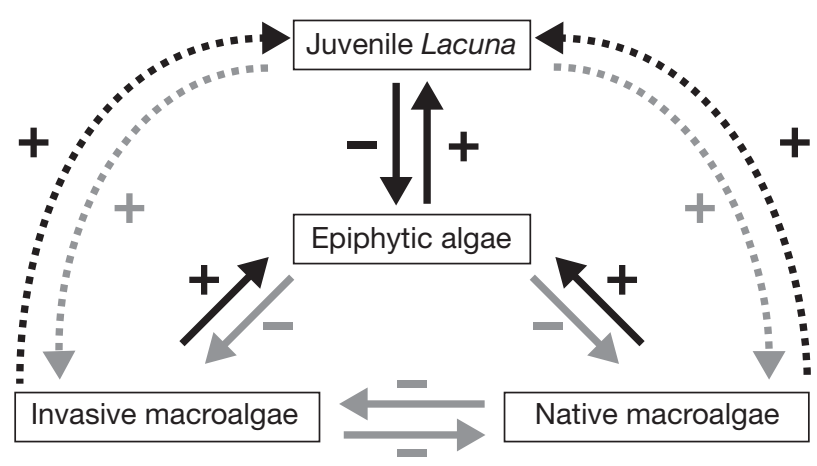

Fig. 5. Diagram showing the potential interactions within this community. Arrows point toward the species experiencing the effect from the one causing it. Signs (+/-) indicate the type of effect. Solid lines represent direct effects, while dashed lines represent indirect effects. Black arrows represent effects shown in the present study, while gray arrows represent interactions suggested by previous research

of Lacuna on Codium (Chavanich \& Harris 2002, Schmidt \& Scheibling 2006); however, the areas sampled had low epiphyte abundances. We found that high Neosiphonia abundance was positively correlated with high Lacuna abundance during the summer (Fig. 4), even at sites where Lacuna and epiphyte abundances were much lower (Jones 2007). In addition, Lacuna preferentially consumed another highly abundant epiphyte, Ceramium (see 'Results - Lacuna surveys and feeding preferences'). Thus, our data suggest that host algal species may facilitate Lacuna by providing food and/or habitat.

\section{SUMMARY}

Many recent studies have examined the importance of positive interactions between species over small time scales or within a few trophic levels (see reviews by Stachowicz 2001, Bruno et al. 2003, Bruno et al. 2005). However, a broad view of these interactions is needed to enhance and modify our understanding of diversity-invasion relationships (Bulleri et al. 2008). As trophic levels are skewed by increasing forces on top predators by humans and high introduction rates of producers and primary consumers (Duffy 2003, Byrnes et al. 2007), the indirect interactions among habitat-modifying invaders and intermediate trophic levels may become important for regulating community dynamics and species diversity. Our findings emphasize the need to investigate new, speciesspecific positive interactions arising from species invasions, as well as the importance of examining the synergistic effects of these interactions across seasons and trophic levels.
Acknowledgements. We thank E. Preisser, S. Nixon, and 3 anonymous reviewers for helpful comments. E. Blair, J. Burkhardt, C. Donahue, E. Field, C. Viall, V. Scialabba, and M. Thompson provided field and laboratory assistance. C. Schneider and J. Foertch provided assistance with epiphyte identification. E.J. was supported by funding from The Sounds Conservancy, URI Office of the Provost, and the URI Department of Biological Sciences. Additional funding was provided by Rhode Island Sea Grant and the Rhode Island Department of Environmental Management to C.T. This work was carried out as partial fulfillment of the requirements for a MS degree by E.J.

\section{LITERATURE CITED}

Amade P, Lemee R (1998) Chemical defence of the Mediterranean alga Caulerpa taxifolia: variations in caulerpenyne production. Aquat Toxicol 43:287-300

Anderson MJ (2001) A new method for non-parametric multivariate analysis of variance. Austral Ecol 26:32-46

> Bakus GJ, Targett NM, Schulte B (1986) Chemical ecology of marine organisms: an overview. J Chem Ecol 12:951-987

Barbàra I, Cremades J (2004) Grateloupia lanceola versus Grateloupia turuturu (Gigartinales, Rhodophyta) en la Penìnsula Ibèrica. An Jardin Botanico Madr 1979 61: 103-118

Bates CR (2009) Host taxonomic relatedness and functionalgroup affiliation as predictors of seaweed-invertebrate epifaunal associations. Mar Ecol Prog Ser 387:125-136

Bouck GB, Morgan E (1957) The occurrence of Codium in Long Island waters. Bull Torrey Bot Club 84:384-387

Bouma TJ, De Vries MB, Low E, Peralta G, Tanczos IC, van de Koppel J, Herman PMJ (2005) Trade-offs related to ecosystem engineering: a case study on stiffness of emerging macrophytes. Ecology 86:2187-2199

Bruno JF, Bertness MD (2001) Habitat modification and facilitation in benthic marine communities. In: Bertness MD, Gaines SD, Hay ME (eds) Marine community ecology. Sinauer Associates, Sunderland, MA, p 201-218

> Bruno JF, Stachowicz JJ, Bertness MD (2003) Inclusion of facilitation into ecological theory. Trends Ecol Evol 18:119-125

Bruno JF, Fridley JD, Bromberg KD, Bertness MD (2005) Insights into biotic interactions from studies of species invasions. In: Sax DF, Stachowicz JJ, Gaines SD (eds) Species invasions: insights into ecology, evolution, and biogeography. Sinauer Associates, Sunderland, MA

Bulleri F, Bruno JF, Benedetti-Cecchi L (2008) Beyond competition: incorporating positive interactions between species to predict ecosystem invasibility. PLoS Biol 6:e162 doi:10.1371/journal.pbio.0060162

Buschbaum C, Chapman A, Saier B (2006) How an introduced species can affect epibiota diversity in different coastal systems. Mar Biol 148:743-754

Byrnes JE, Reynolds PL, Stachowicz JJ (2007) Invasions and extinctions reshape coastal marine food webs. PLoS ONE 2:e295

Casas G, Scrosati R, Luz Piriz M (2004) The invasive kelp Undaria pinnatifida (Phaeophyceae, Laminariales) reduces native seaweed diversity in Nuevo Gulf (Patagonia, Argentina). Biol Invasions 6:411-416

Cattaneo A (1983) Grazing on epiphytes. Limnol Oceanogr 28:124-132

> Chavanich S, Harris LG (2002) The influence of macroalgae on seasonal abundance and feeding preference of a subtidal snail, Lacuna vincta (Montagu) (Littorinidae) in the Gulf of Maine. J Molluscan Stud 68:73-78 
Choi HG, Kim MS, Guiry MD, Saunders GW (2001) Phylogenetic relationships of Polysiphonia (Rhodomelaceae, Rhodophyta) and its relatives based on anatomical and nuclear small-subunit rDNA sequence data. Can J Bot 79: $1465-1476$

> Clarke KR (1993) Non-parametric multivariate analyses of changes in community structure. Aust J Ecol 18:117-143

Crooks JA (1998) Habitat alteration and community-level effects of an exotic mussel, Musculista senhousia. Mar Ecol Prog Ser 162:137-152

Crooks JA (2002) Characterizing ecosystem-level consequences of biological invasions: the role of ecosystem engineers. Oikos 97:153-166

D'Antonio C (1985) Epiphytes on the rocky intertidal red alga Rhodomela larix (Turner) C. Agardh: Negative effects on the host and food for herbivores? J Exp Mar Biol Ecol 86: $197-218$

Davis AR, Targett NM, McConnell OJ, Young CM (1989) Epibiosis of marine algae and benthic invertebrates: natural products chemistry and other mechanisms inhibiting settlement and overgrowth. In: Scheuer PJ (ed) Bioorganic marine chemistry, Vol 3. Springer, Berlin, p 85-114

> Dawes CJ, Teasdale BW, Friedlander M (2000) Cell wall structure of the agarophytes Gracilaria tikvahiae and G. cornea (Rhodophyta) and penetration by the epiphyte Ulva lactuca (Chlorophyta). J Appl Phycol 12: 567-576

> Dayton P (1971) Competition, disturbance, and community organization: the provision and subsequent utilization of space in a rocky intertidal community. Ecol Monogr 41: 351-389

Dayton PK (1972) Toward an understanding of community resilience and the potential effects of enrichment to the benthos of McMurdo Sound, Antarctica. In: Parker BC (ed) Proc Colloquium Conserv Problems Antarctica. Allen Press, Lawrence, KS, p 81-95

> Dean RL, Connell JH (1987) Marine invertebrates in an algal succession. III. Mechanisms linking habitat complexity with diversity. J Exp Mar Biol Ecol 109:249-273

Duffy JE (1990) Amphipods on seaweeds: Partners or pests? Oecologia 83:267-276

Duffy JE (2003) Biodiversity loss, trophic skew and ecosystem functioning. Ecol Lett 6:680-687

Filion-Myklebust C, Norton T (1981) Epidermis shedding in the brown seaweed Ascophyllum nodosum (L.) Le Jolis, and its ecological significance. Mar Biol Lett 2:45-51

Grabowski JH, Hughes AR, Kimbro DL, Dolan MA (2005) How habitat setting influences restored oyster reef communities. Ecology 86:1926-1935

Harlin MM, Villalard-Bohnsack M (2001) Seasonal dynamics and recruitment strategies of the invasive seaweed Grateloupia doryphora (Halymeniaceae, Rhodophyta) in Narragansett Bay and Rhode Island Sound, Rhode Island, USA. Phycologia 40:468-474

> Harris LG, Tyrell MC (2001) Changing community states in the Gulf of Maine: synergisms between invaders, overfishing, and climate change. Biol Invasions 3:9-21

Hellio C, Simon-Colin C, Clare AS, Deslandes E (2004) Isethionic acid and floridoside isolated from the red alga, Grateloupia turuturu, inhibit settlement of Balanus amphitrite cyprid larvae. Biofouling 20:139-145

Idjadi JA, Edmunds PJ (2006) Scleractinian corals as facilitators for other invertebrates on a Caribbean reef. Mar Ecol Prog Ser 319:117-127

Jernakoff P, Brearley A, Nielsen J (1996) Factors affecting grazer-epiphyte interactions in temperate seagrass meadows. Oceanogr Mar Biol Annu Rev 34:109-162
Jones E (2007) Impacts of habitat-modifying invasive macroalgae on epiphytic algal communities. MS thesis, University of Rhode Island, Kingston, RI

Karez R, Engelbert S, Sommer U (2000) 'Co-consumption' and 'protective coating': two new proposed effects of epiphytes on their macroalgal hosts in mesograzerepiphyte-host interactions. Mar Ecol Prog Ser 205:85-93

Levin PS, Coyer JA, Petrik R, Good TP (2002) Community effects of nonindigenous species on temperate rocky reefs. Ecology 83:3182-3193

> Lohse DP (1993) The importance of secondary substratum in a rocky intertidal community. J Exp Mar Biol Ecol 166:1-17

> Lubchenco J (1980) Algal zonation in the New England rocky intertidal community: an experimental analysis. Ecology 61:333-344

Mathieson AC, Dawes CJ, Harris LG, Hehre EJ (2003) Expansion of the Asiatic green alga Codium fragile subsp. tomentosoides in the Gulf of Maine. Rhodora 105:1-53

Mattson WJ (1980) Herbivory in relation to plant nitrogen content. Annu Rev Ecol Syst 11:119-161

> Menge BA (1976) Organization of the New England rocky intertidal community: role of predation, competition, and environmental heterogeneity. Ecol Monogr 46:355-393

Moss BL (1982) The control of epiphytes by Halidrys siliquosa (L.) Lynbg. (Phaeophyta, Cystoseiraceae). Phycologia 21: 185-191

> Norton TA, Benson MR (1983) Ecological interactions between the brown seaweed Sargassum muticum and its associated fauna. Mar Biol 75:169-177

> Orav-Kotta H, Kotta J (2004) Food and habitat choice of the isopod Idotea baltica in the northeastern Baltic Sea. Hydrobiologia 514:79-85

Orth RJ, Heck KL (1980) Structural components of eelgrass (Zostera marina) meadows in the lower Chesapeake Bay: fishes. Estuaries 3:278-288

> Orth RJ, van Montfrans J (1984) Epiphyte-seagrass relationships with an emphasis on the role of micrograzing: a review. Aquat Bot 18:43-69

> Parker IM, Simberloff D, Lonsdale WM, Goodell K, Wonham M (1999) Impact: toward a framework for understanding the ecological effects of invaders. Biol Invasions 1:3-19

Paul VJ, Puglisi MP, Ritson-Williams R (2006) Marine chemical ecology. Nat Prod Rep 23:153-180

Pavia H, Carr H, Aberg P (1999) Habitat and feeding preferences of crustacean mesoherbivores inhabiting the brown seaweed Ascophyllum nodosum (L.) Le Jol. and its epiphytic macroalgae. J Exp Mar Biol Ecol 236:15-32

> Posey MH (1988) Community changes associated with the spread of an introduced seagrass, Zostera japonica. Ecology 69:974-983

Rice WR (1990) A consensus combined p-value test and the family-wide significance of component tests. Biometrics 46:303-308

> Rodriguez LF (2006) Can invasive species facilitate native species? Evidence of how, when, and why these impacts occur. Biol Invasions 8:927-939

> Russell G, Veltkamp CJ (1984) Epiphyte survival on skinshedding macrophytes. Mar Ecol Prog Ser 18:149-153

> Sanchez I, Fernandez C, Arrontes J (2005) Long-term changes in the structure of intertidal assemblages after invasion by Sargassum muticum (Phaeophyta). J Phycol 41:942-949

Sanders NJ, Gotelli NJ, Heller NE, Gordon DM (2003) Community disassembly by an invasive species. Proc Natl Acad Sci USA 100:2474-2477

Scheibling RE, Gagnon P (2006) Competitive interactions between the invasive green alga Codium fragile ssp. 
tomentosoides and native canopy-forming seaweeds in Nova Scotia (Canada). Mar Ecol Prog Ser 325:1-14

Schmidt AL, Scheibling RE (2006) A comparison of epifauna and epiphytes on native kelps (Laminaria species) and an invasive alga (Codium fragile ssp. tomentosoides) in Nova Scotia, Canada. Bot Mar 49:315-330

Schmitt TM, Hay ME, Lindquist N (1995) Constraints on chemically mediated coevolution: multiple functions for seaweed secondary metabolites. Ecology 76:107-123

Seed R, O'Connor RJ (1981) Community organization in marine algal epifaunas. Annu Rev Ecol Syst 12:49-74

Sieburth JM, Tootle JL (1981) Seasonality and microbial fouling on Ascophyllum nodosum (L.) Lejol., Fucus vesiculosus L., Polysiphonia lanosa (L.) Tandy and Chondrus crispus Stackh. J Phycol 17:57-64

Southgate T (1982) A comparative study of Lacuna vincta and Lacuna pallidula (Gastropoda: Prosobranchia) in littoral algal turfs. J Molluscan Stud 48:302-309

Stachowicz JJ (2001) Mutualism, facilitation, and the structure of ecological communities. BioScience 51:235-245

Stachowicz JJ, Hay ME (1999) Mutualism and coral persistence: the role of herbivore resistance to algal chemical defense. Ecology 80:2085-2101

Stachowicz JJ, Whitlatch RB (2005) Multiple mutualists provide complementary benefits to their seaweed host. Ecology 86:2418-2427

Steinberg PD, De Nys R, Kjelleberg S (2002) Chemical cues for surface colonization. J Chem Ecol 28:1935-1951

Stewart JG (1982) Anchor species and epiphytes in intertidal algal turf. Pac Sci 36:45-59

Strong JA, Dring MJ, Maggs CA (2006) Colonisation and modification of soft substratum habitats by the invasive macroalga Sargassum muticum. Mar Ecol Prog Ser 321:87-97

Trowbridge CD (1999) An assessment of the potential spread and options for control of the introduced green macroalga Codium fragile ssp. tomentosoides on Australian shores. Centre for Research on Introduced Marine Pests, Hobart, Tasmania

Underwood AJ (1997) Experiments in ecology: their logical design and interpretation using analysis of variance. Cambridge University Press, Cambridge

Editorial responsibility: Lisandro Benedetti-Cecchi, Pisa, Italy
Villalard-Bohnsack M (2003) Illustrated key to the seaweeds of New England. Rhode Island Natural History Survey, Kingston, RI

Villalard-Bohnsack M, Harlin MM (1997) The appearance of Grateloupia doryphora (Halymeniaceae, Rhodophyta) on the northeast coast of North America. Phycologia 36: $324-328$

Villalard-Bohnsack M, Harlin MM (2001) Grateloupia doryphora (Halymeniaceae, Rhodophyta) in Rhode Island waters (USA): geographical expansion, morphological variations and associated algae. Phycologia 40: $372-380$

Villalard-Bohnsack M, Peckol P, Harlin MM (1988) Marine macroalgae of Narragansett Bay and adjacent sounds. In: Sheath RG, Harlin MM (eds) Freshwater and marine plants of Rhode Island. Kendall/Hunt Publishing Company, Dubuque, IA

> Wernberg T, Thomsen MS, Staehr PA, Pedersen MF (2004) Epibiota communities of the introduced and indigenous macroalgal relatives Sargassum muticum and Halidrys siliquosa in Limfjorden (Denmark). Helgol Mar Res 58: $154-161$

> Wikström SA, Kautsky L (2004) Invasion of a habitat-forming seaweed: effects on associated biota. Biol Invasions 6: $141-150$

Wikström SA, Pavia H (2004) Chemical settlement inhibition versus post-settlement mortality as an explanation for differential fouling of two congeneric seaweeds. Oecologia 138:223-230

Wilcove DS, Rothstein D, Dubow J, Phillips A, Losos E (1998) Quantifying threats to imperiled species in the United States. BioScience 48:607-615

Williams GA, Seed R (1992) Interactions between macrofaunal epiphytes and their host algae. In: John DM, Hawkins $\mathrm{SJ}$, Price JH (eds) Plant-animal interactions in the marine benthos, Vol 46. Systematics Association Special, Clarendon Press, Oxford, p 189-211

Williamson M (1996) Biological invasions. Chapman \& Hall, New York

Wilson L (1978) Epiphytes on Codium fragile (Chlorophyceae). MS thesis, University of Rhode Island, Kingston, RI

Submitted: September 15, 2008; Accepted: October 28, 2009 Proofs received from author(s): February 1, 2010 